\title{
Reduced postpartum hemorrhage after implementation of active management of the third stage of labor in rural Honduras
}

\author{
Lisa Kane Low ${ }^{\text {a,b, } *}$, Joanne Motino Bailey ${ }^{c}$, Emma Sacks ${ }^{d}$, Chayla Robles ${ }^{\text {b }}$, Lilian Medina ${ }^{\text {e }}$ \\ a Women's Studies Department, University of Michigan, Ann Arbor, USA \\ b School of Nursing, University of Michigan, Ann Arbor, USA \\ c University of Michigan Health System, Ann Arbor, USA \\ d Department of International Health, Johns Hopkins School of Public Health, Baltimore, USA \\ e Negrito Health Center, Yoro, Honduras
}

\section{A R T I C L E I N F O}

\section{Article history:}

Received 30 March 2012

Received in revised form 5 July 2012

Accepted 15 August 2012

\section{Keywords:}

Active management of third stage labor

AMTSL

Honduras

Intramuscular oxytocin

Postpartum hemorrhage

Safe Motherhood

Skilled birth attendants

\begin{abstract}
A B S T R A C T
Objective: To assess outcomes after auxiliary nurses were trained and given resources to use active management of the third stage of labor (AMTSL) for all women giving birth in a low-resource, low-risk, rural, public birth center setting in northern rural Honduras. Methods: Auxiliary nurses received training on estimation of blood loss before the preintervention phase of the study (July 2004 through April 2005) and AMTSL, including use of intramuscular oxytocin, and estimation of blood loss prior to the intervention phase (July 2007 through June 2008). Preintervention and intervention data on use of oxytocin, blood loss postpartum, hemorrhage rates, and management interventions were collected and compared. Results: After nurses received training on AMTSL using intramuscular oxytocin, the use of intramuscular oxytocin during the third stage of labor increased from $63.8 \%$ to $96.5 \%$. Postpartum hemorrhage rates decreased from $14.8 \%$ to $5.9 \%(P=0.001)$. Use of intrapartum oxytocin, which can have adverse effects, also increased: from $6.1 \%$ to 22.7\% $(P<0.001)$. Conclusion: Training auxiliary nurses to perform AMTSL using oxytocin in this birth center setting was effective in reducing the rate of postpartum hemorrhage; however, increased use of intrapartum oxytocin may be an unintended outcome of the increased accessibility of oxytocin.

(C) 2012 International Federation of Gynecology and Obstetrics. Published by Elsevier Ireland Ltd. All rights reserved.
\end{abstract}

\section{Introduction}

Postpartum hemorrhage (PPH) is the leading cause of maternal morbidity and mortality worldwide and accounts for $25 \%$ of all maternal deaths [1]. Active management of the third stage of labor (AMTSL) is an evidence-based strategy to reduce the incidence of uterine atony, which is the primary cause of PPH [2]. Components of AMTSL include administration of uterotonic medications to the mother within 1 minute after delivery, use of controlled cord traction in delivery of the placenta, and uterine massage following placental delivery [1]. The use of AMTSL can reduce the risk of PPH (blood loss greater than $500 \mathrm{~mL}$ ) and severe PPH (blood loss greater than $1000 \mathrm{~mL}$ ) and decrease the need for blood transfusion or additional uterotonic medications [2].

The International Federation of Gynecology and Obstetrics (FIGO) and the International Confederation of Midwives (ICM) issued a joint statement in 2003 and reinforced it in 2006, recommending routine use of AMTSL in all vaginal births as the optimal standard of maternity care in all settings [1]. However, implementation of AMTSL requires training and resources [3]. Implementation challenges in low-resource

\footnotetext{
* Corresponding author at: 400 N, Ingalls, Suite 3320, Ann Arbor, MI 48109, USA. Tel.: + 17346470136

E-mail address: kanelow@umich.edu (L.K. Low).
}

countries include variations in curricula, conflicting policies, insufficient training, and variable access to uterotonic agents.

Honduras currently has a maternal mortality rate of 110 per 100000 live births [4], which represents a decrease from 186 per 100000 during the 1990s [5]. Successful efforts to reduce maternal mortality in Honduras have focused on increasing the number of births attended by skilled birth attendants in hospitals and free-standing birth centers [6]. In 2004, the Honduran Ministry of Public Health officially endorsed the universal application of AMTSL as a standard of maternity care in all settings as a means of continuing to reduce maternal morbidity and mortality.

Clínica Materno-Infantíl (CMI) is a free-standing, public birth center located in northern rural Honduras in the district of Morazán. The clinic offers pregnancy and delivery services to low-risk women from the surrounding area, including rural communities located as much as 4 hours away. It is the site of previous maternal health research [7], is staffed by auxiliary nurses, and is administratively supervised by a professional nurse and a general medicine physician who are normally available on-call. The auxiliary nurses attend most births alone without other immediate help. The Honduran auxiliary nurse curriculum is a 1-year program that includes prenatal care knowledge and experience, but not care during childbirth. Prior to staffing at $\mathrm{CMI}$, an auxiliary nurse must observe and perform supervised births 
for 1 month in a public hospital. Following this experience, the auxiliary nurse works with an experienced auxiliary nurse or the professional nurse at CMI in a final process of orientation prior to attending births independently.

The aim of the present study was to assess the impact of implementing AMTSL in a low-resource, government-funded birth center staffed by auxiliary nurses in rural northern Honduras.

\section{Materials and methods}

This project was part of a larger project examining transitions in reproductive and maternity care for women in Morazán, Yoro, Honduras. The study was approved by the Institutional Review Board at the University of Michigan, officials at CMI, and the local Ministry of Public Health in Honduras. In July 2004, the research team trained professional nurses and auxiliary nurses on the causes and management of PPH (e.g. fundal massage, bimanual compression). Participants were instructed in methods to estimate blood loss for all births that occurred at the CMI. The goal of this initial study was to determine the rate of PPH and to begin to quantify blood loss post delivery for women cared for by auxiliary nurses.

In the preintervention phase, the auxiliary nurses were trained to collect all postpartum vaginal blood in a stainless steel bowl below the delivery table. The bowl included markings that noted estimated blood volume, which were also depicted on a poster in the birth room. The nurses visually quantified the blood loss based on the markings and also included the amount of lost blood present on the birth table and in the drapes. The methods used for this process are described in further detail elsewhere [7]. The staff completed two repeated trainings for estimating blood loss over the course of the study period to confirm skill development and maintenance. The auxiliary nurses did not receive training on the specific use of AMTSL at this time owing to constrained resources at CMI.

In June 2007, a second training session was conducted to reinforce the lessons of the initial training and add instruction on the 3 steps of AMTSL, including use of intramuscular oxytocin immediately following delivery. In the intervention phase, the step of measuring blood loss using a graduated cylinder was added. The nurse also documented the estimated amount of additional blood not captured in the bowl, including blood on the floor, drapes, and delivery table. These measurements were used for a total blood loss value. During this second phase, oxytocin was made available for every woman giving birth at CMI. Following delivery, basic demographics, total blood loss (measured blood loss plus estimated blood unable to be measured), labor management practices, intravenous catheter and fluid use during labor and postpartum, oxytocin postpartum, and PPH rates were recorded by the auxiliary nurse who attended the birth.

A local Honduran research assistant collected the forms daily and entered the data into an SPSS electronic spreadsheet. Any unclear responses on the data form were clarified with the birth attendant. Empirical data from the first and second phases were combined and analyzed using SPSS version 15 (IBM, Armonk, NY, USA) for descriptive statistics, as well as the $t$ test and $\chi^{2}$ test. $P<0.05$ was considered statistically significant.

\section{Results}

The first phase-the preintervention time period-extended from July 27, 2004, through April 30, 2005, and included 229 women who gave birth at CMI. The second phase-the intervention period-extended from July 2, 2007, through June 30, 2008, and included 339 women. Both time periods had similar patient populations. Mean age overall was approximately 23 years in both phases. However, parity was slightly lower in the second time period ( 1.5 vs $2.0 ; P=0.016$ ). There were no maternal deaths at CMI during either study period.
Table 1

Comparison of preintervention and intervention group demographics and selected outcomes of care. $^{\mathrm{a}}$

\begin{tabular}{|c|c|c|c|}
\hline & $\begin{array}{l}\text { Preintervention } \\
(\mathrm{n}=229)\end{array}$ & $\begin{array}{l}\text { Intervention } \\
(\mathrm{n}=339)\end{array}$ & $P$ value \\
\hline \multicolumn{4}{|l|}{ Demographics } \\
\hline Age, y & $23.4 \pm 6.8$ & $23.1 \pm 6.5$ & 0.505 \\
\hline Parity & $2 \pm 2.5$ & $1.5 \pm 2.1$ & 0.016 \\
\hline \multicolumn{4}{|l|}{ Labor management } \\
\hline Intravenous fluid used during labor & $9(3.9)$ & $77(22.7)$ & $<0.001$ \\
\hline Labor augmentation with oxytocin & $14(6.1)$ & $77(22.7)$ & $<0.001$ \\
\hline Episiotomy performed & $55(24.0)$ & $82(24.2)$ & \\
\hline \multicolumn{4}{|l|}{ PPH prevention and management } \\
\hline Estimated blood loss, $\mathrm{mL}$ & $355 \pm 271$ & $247 \pm 186$ & $0.009^{b}$ \\
\hline Oxytocin administered postpartum & $146(63.8)$ & $327(96.5)$ & $<0.001^{\mathrm{c}}$ \\
\hline $\begin{array}{l}\text { PPH estimated blood loss } \\
\text { (revised for } 07-08), \geq 500 \mathrm{~mL}\end{array}$ & $34(14.8)$ & $20(5.9)$ & $0.001^{\mathrm{c}}$ \\
\hline IV placed postpartum & $20(8.7)$ & $35(10.3)$ & $0.565^{\mathrm{c}}$ \\
\hline Methergine use & $2(0.9)$ & $0(0)$ & \\
\hline
\end{tabular}

Abbreviations: $\mathrm{PPH}$, postpartum hemorrhage; IV, intravenous.

a Values are given as mean \pm SD and number (percentage) unless otherwise indicated.

b $t$ test with unequal variances.

c $\chi^{2}$ test.

Labor management practices are presented in Table 1. Episiotomy rates were stable. There was a significant marked increase in the use of oxytocin during labor from 14 to 77 cases ( $6.1 \%$ vs $22.1 \% ; P<0.001$ ).

Following training and implementation of AMTSL, use of oxytocin immediately following birth increased (63.8\% vs 96.5\% $P<0.001)$, estimated blood loss (EBL) decreased $(355 \pm 271 \mathrm{~mL}$ vs $247 \pm 186 \mathrm{~mL} ; P=0.009)$, and $\mathrm{PPH}$ rates decreased $(14.8 \%$ vs $5.9 \%$, $P=0.001$ ) (Table 1).

There were 6 cases of severe PPH in the preintervention period, accounting for $2.6 \%$ of the overall cases. The range of severe blood loss was 1000-3000 mL. In the intervention phase there were 4 cases of severe PPH, accounting for $1.2 \%$ of the cases where the documented blood loss was $1250-1500 \mathrm{~mL}$. There was no statistical difference between these 2 rates of severe hemorrhage $(P=0.185)$. Intravenous catheter placement over the 2 time periods was unchanged ( $8.7 \%$ vs $10.3 \%, P=0.565)$.

Women who received intrapartum oxytocin, a labor augmentation technique, had a higher mean EBL compared with women who did not receive oxytocin intraparum $(319 \pm 250 \mathrm{~mL}$ vs $222 \pm 163 \mathrm{~mL}$; $P=0.007$ ) (Table 2). In addition, an increased rate of PPH was observed for women who underwent labor augmentation compared with those who did not ( $15.3 \%$ vs $3.4 \% ; P<0.001)$.

In a post hoc analysis based on the unexpected finding of an increased use of intrapartum oxytocin, there was no statistically significant difference in the mean length of labor for primiparas and multiparas when analyzed separately based on those who received intrapartum oxytocin compared with those who did not $(P=0.055)$. Total time in labor was not correlated with having a higher EBL (Pearson $=0.038, P=0.493$ ), and after controlling for length of labor using regression modeling, the use of intrapartum oxytocin was significantly correlated with a higher $\operatorname{EBL}(P=0.001)$.

Table 2

Oxytocin administration for augmentation of labor in the intervention group. ${ }^{a}$

\begin{tabular}{lcll}
\hline & $\mathrm{n}=334$ & $\begin{array}{l}\text { Estimated } \\
\text { blood } \\
\text { loss, } \mathrm{mL}\end{array}$ & $\begin{array}{l}\text { Postpartum } \\
\text { hemorrhage }\end{array}$ \\
\hline Oxytocin administered intrapartum & 72 & $319 \pm 250$ & $11(15.3)$ \\
Oxytocin NOT administered intrapartum & 262 & $\begin{array}{l}222 \pm 163 \\
P=0.007^{\mathrm{b}}\end{array}$ & $\begin{array}{l}9(3.4) \\
P<0.001^{\mathrm{c}}\end{array}$ \\
\hline
\end{tabular}

${ }^{a}$ Values are given as mean \pm SD and number (percentage) unless otherwise indicated.

b $t$ test unequal means.

c $\chi^{2}$ test. 


\section{Discussion}

The results of the present study indicate that PPH can be reduced when auxiliary nurses are instructed on the use of AMTSL in a low-resource birth center setting. Almost all women received intramuscular administration of oxytocin postpartum as part of AMTSL (96.5\%). This compared favorably to the preintervention phase when oxytocin was not used as a component of AMTSL and was intermittently available. With the intervention, women experienced a reduction in PPH of almost 9\%. This reduction in the incidence of PPH was combined with an overall reduction in mean EBL of $108 \mathrm{~mL}$ for the women giving birth at CMI. Although the clinical implications of this reduction in blood loss overall were not assessed, other investigators have argued that a reduction in EBL provides positive benefits, particularly in low-resource settings where high rates of anemia are prevalent [8]. Overall, the present study indicates that auxiliary nurses in a low-resource setting can be effectively trained in the use of AMTSL to reduce the incidence of PPH. The introduction of this intervention appears to produce positive results for the women who seek childbirth care at CMI.

The use of AMTSL has been evaluated in hospital settings in Honduras, and researchers found only a $20 \%$ use of properlyadministered uterotonics for the purpose of AMTSL in facility-based births [3]. The results of the present study compare favorably with the results of this investigation in promoting increased use of AMTSL by auxiliary nurses at CMI. Despite the 2004 Honduran Ministry of Public Health's endorsement of the use of AMTSL as the standard of care, according to a 2006 study published by the United States Agency for International Development (USAID) and the Prevention of Post-Partum Hemorrhage International project, AMTSL was not included in any of the nursing school curricula and instruction on its use was inconsistently provided during in-service education in facilities where maternity services were provided [3]. Thus, the need for a standardized curriculum and approach to training is an important step in increasing the correct and consistent use of AMTSL.

Despite the positive results of increased use of AMTSL at CMI, there were consequences to having increased access to oxytocin in this setting. With the rise in postpartum oxytocin use, there was also a rise in use during the first stage of labor. This increase in use was not correlated with increased length of labor or any other risk factor that would medically justify its use, particularly in a low-risk birth center where continuous fetal assessment is not possible. The results demonstrated that with the rise in oxytocin use during the first stage of labor, there was a significant increase in mean blood loss for participants. This increased blood loss was combined with a significantly increased risk for PPH. The usual management and use of intrapartum oxytocin at CMI requires that a physician is present, but physician involvement was not evident in the cases in the second phase of the study. Of note, when the results of this investigation were presented to the staff at CMI, they immediately responded and reaffirmed that the use of oxytocin in labor was an indication for transfer to a hospital setting and/or having a physician on site, which supported the standard of practice.

The risk of overutilization of oxytocin during the intrapartum phase has been identified by other investigators who evaluated the implementation of AMTSL where oxytocin was the uterotonic agent of choice $[9,10]$. However, it is unclear if the use of other uterotonic agents would have potentially reduced or eliminated the risk associated with oxytocin. Alternative uterotonic agents such as misoprostol may not have presented as great a risk for use during labor, but the nurses were not familiar with these agents as labor induction or augmentation agents. Because the nurses had experience with oxytocin use during their hospital training, it is possible that they were more confident in using it in the birth center, even though it was against practice standards. The present study included a series of educational seminars that focused on presenting the results of the project over time. Because of this, the implications of increased blood loss and hemorrhage with the increased use of oxytocin in the intrapartum period could be presented, which resulted in staff awareness and cessation of the practice.

Implementation of AMTSL in low-resource settings like CMI has the potential to reduce the incidence of PPH, but is not without challenges. Fundamental barriers to the use of oxytocin in AMTSL are the limited availability of needles and syringes and staffing challenges [11]. Levold [9] recommended ways to avoid the risk of over use of oxytocin, including instruction on appropriate and inappropriate use of oxytocin, evidence-based clinical guidelines, and research to document rates of induction/augmentation. These steps were put into place during the present investigation at CMI, yet the assumption that current labor augmentation policies would be maintained when access to oxytocin became routine was not realized. When new practice patterns are introduced, additional supervision has been shown to be useful for the reduction of excess use of uterotonics [3], but this step was not in place at CMI. Finally, the implications of the use of intrapartum oxytocin include not only the finding of increased incidence of $\mathrm{PPH}$, but also may include reduced effectiveness of agents used to treat PPH following intrapartum oxytocin use [12].

The present study is limited in its generalizability to other sites and settings using other types of skilled birth attendants who may have additional education regarding the risks and appropriate uses of oxytocin during childbirth. The potential for bias within the conduct of the trial was considered given the unblinded nature of the design and documentation of EBL by the same staff member who provided care. In the context of the low-resource setting of $\mathrm{CMI}$, the methods and equipment used for the present study were consistent with what was available on the site and sustainable beyond the actual trial. In general, estimating blood loss visually usually results in underrepresentation of the total compared with other methods $[13,14]$. Thus, the preintervention phase EBLs risk being an underrepresentation of the total EBL. The addition of the graduated cylinder for measuring blood loss in the intervention phase should have refined the measurements and removed the risk for bias in favor of AMTSL.

Finally, a potential secular trend influencing the data could have been the improved skill of the birth attendants overall in PPH management outside of the use of AMTSL. Based on observations by the research team and independent observations of student midwives from the USA who were present at CMI during the study period, there were no obvious changes in practice patterns other than the application of increased use of oxytocin; however, observers were only present over 12 weeks of the study period at intervals of 2-3 weeks at a time. Performing AMTSL increased staff work effort, which could arguably be a potential risk for bias against its use. Despite increased staff effort, during the observation phases the staff was observed conducting EBL estimations as instructed.

In summary, auxiliary nurses at $\mathrm{CMI}$ in Honduras were able to effectively implement AMTSL following training, which contributed to reduced estimated PPH rates and reduced mean postpartum estimated blood loss. However, with the increased availability of oxytocin in the birth center, the rate of intrapartum oxytocin use also increased, resulting in overuse that has the potential to conflict with the improvements that can be achieved with implementation of AMTSL alone. Continued education about the risks and appropriate use of oxytocin, including presentations of outcome data during implementation of new practices, combined with appropriate supervisory vigilance during the process can potentially mitigate this risk.

\section{Acknowledgments}

Funding was provided by the Institute for Research on Women and Gender, University of Michigan; the Global Reach Program in the School of Medicine, the Rackham Summer Interdisciplinary 
Institute, University of Michigan; and the School of Nursing, Office of International Affairs, University of Michigan.

\section{Conflict of interest}

The authors declare no conflicts of interest.

\section{References}

[1] International Confederation of Midwives, International Federation of Gynecology and Obstetrics. Joint statement: management of the third stage of labour to prevent post-partum haemorrhage. J Midwifery Womens Health 2004;49(1):76-7.

[2] Begley CM, Gyte GM, Devane D, McGuire W, Weeks A. Active versus expectant management for women in the third stage of labour. Cochrane Database Syst Rev 2011(11):CD007412.

[3] Stanton C, Armbruster D, Knight R, Ariawan I, Gbangbade S, Getachew A, et al. Use of active management of the third stage of labour in seven developing countries. Bull World Health Organ 2009;87(3):207-15.

[4] World Health Organization, United Nations Children's Fund, United Nations Population Fund, The World Bank. Trends in maternal mortality: 1990 to 2008. http://whqlibdoc.who.int/publications/2010/9789241500265_eng.pdf; 2010.

[5] Danel I. Maternal mortality reduction, Honduras, 1990-1997: a case study. Washington, DC: World Bank; 1998
[6] Shiffman J, Stanton C, Salazar AP. The emergence of political priority for safe motherhood in Honduras. Health Policy Plan 2004;19(6):380-90.

[7] Low LK, Bailey JM, Sacks E, Medina L, Piñeda HO. Postpartum hemorrhage prevention: a case study in northern rural Honduras. J Midwifery Womens Health 2008;53(1): e1-6.

[8] McDonald S. Management of the third stage of labor. J Midwifery Womens Health 2007;52(3):254-61

[9] Lovold A, Stanton C, Armbruster D. How to avoid iatrogenic morbidity and mortality while increasing availability of oxytocin and misoprostol for PPH prevention? Int J Gynecol Obstet 2008;103(3):276-82.

[10] Flandermeyer D, Stanton C, Armbruster D. Uterotonic use at home births in lowincome countries: a literature review. Int J Gynecol Obstet 2010;108(3):269-75.

[11] Motino J, Medina L, Kane L. Prevencion de la hemorragia posparto (Postpartum hemorrhage prevention). Horiz Enferm 2010;21(1):37-43.

[12] Blum J, Winikoff B, Raghavan S, Dabash R, Ramadan MC, Dilbaz B, et al. Treatment of post-partum haemorrhage with sublingual misoprostol versus oxytocin in women receiving prophylactic oxytocin: a double-blind, randomised, non-inferiority trial. Lancet 2010;375(9710):217-23.

[13] Schorn MN. Measurement of blood loss: review of the literature. J Midwifery Womens Health 2010;55(1):20-7.

[14] Patel A, Goudar SS, Geller SE, Kodkany BS, Edlavitch SA, Wagh K, et al. Drape estimation vs. visual assessment for estimating postpartum hemorrhage. Int J Gynecol Obstet 2006;93(3):220-4. 